\title{
Insights into the design, use and implementation of home energy management systems
}

\author{
S.S. Van Dam* \\ Faculty of A rchitecture, \\ Delft University of Technology, \\ Julianalaan 134, 2628 B L D elft, The N etherlands \\ E-mail: s.s.vandam@ tudelft.nl \\ *Corresponding author
}

\section{C.A. Bakker}

Faculty of Industrial Design Engineering,

Delft U niversity of Technology,

Landbergstraat 15, 2628 CE Delft, The N etherlands

E-mail: c.a.bakker@ tudelft.nl

\author{
J.D.M. Van Hal \\ Faculty of A rchitecture, \\ Delft University of Technology, \\ Julianalaan 134, 2628 B L D elft, The Netherlands \\ and \\ Nyenrode Business U niversity, \\ Straatweg 25, 3621 BG Breukelen, The N etherlands \\ E-mail: j.d.m.vanhal@ tudelft.nl
}

\begin{abstract}
Between 2008 and 2011, three different home energy management systems (HEMS) that give feedback on energy consumption were implemented in households in the Netherlands. Home energy management systems are defined as intermediary devices that can visualise, monitor and/or manage domestic gas and/or electricity consumption. Through a series of questionnaires, interviews, focus groups and usability tests, a wide range of knowledge was gathered on factors influencing the effectiveness of the three systems. The resulting insights were structured with the help of a conceptual model, which outlines the various interactions between users, energy monitors, and their social and physical environments. The insights provide a broad spectrum of factors to be considered for the successful design and implementation of home energy management systems. This paper aims to provoke an open discussion to ascertain the value of the different factors and further the development of effective and useful HEM S.
\end{abstract}

Keywords: energy monitor; smart metering; energy conservation; household energy consumption; feedback; usability; design research; home energy management system; insights; implementation. 
Reference to this paper should be made as follows: $V$ an Dam, S.S., Bakker, C.A. and V an Hal, J.D.M. (xxxx) 'Insights into the design, use and implementation of home energy management systems', J. Design Research, Vol. X, No. Y, pp.000-000.

Biographical notes: S.S. V an Dam is currently conducting her PhD research on 'Smart energy management for households' in a joint cooperation between the faculties of Architecture and Industrial Design Engineering. She has a background in Industrial Design Engineering with a Master in Design for Interaction. Her research interest is the effectiveness of home energy management systems (HEMS); how they are incorporated into people's daily lives, and how they affect the choices people make concerning their energy consumption and their homes.

C.A. Bakker is an Assistant Professor at the Faculty of Industrial Design Engineering, with specialisation in Design for Sustainability. Her research focuses on the development of methods, tools and demonstrators for commercially viable products that embrace material durability, sustained use and reuse, and prolonged affective life spans. She has a particular interest in the relationships between sustainability, design and human behaviour.

J.D.M. Van Hal is Professor of Sustainable Building and Development at the Center for Sustainability of Nyenrode Business University. She is also a Professor of Sustainable Housing Transformation at the Faculty of A rchitecture of Delft University of Technology. Her chair focuses on increasing the sustainability of existing housing stocks and neighbourhoods, to ensure they meet ambitious environmental targets and reflect residents' needs.

\section{Introduction}

Home energy management systems (HEM S) are defined as intermediary devices that can visualise, monitor and/or manage domestic gas and/or electricity consumption ( $V$ an Dam et al., 2010). Their main purpose is to give users direct and accessible insight into their energy consumption. This makes them different from smart meters, which are predominantly intended for automatic two-way communication of energy data between the gas or electricity supplier and homes. Smart meters generally need HEMS to give users the intended insight.

HEMS are being given increasing attention both in academia and in commercial enterprises and are much advertised and promoted as 'high potentials' for domestic energy savings. Studies have indeed reported positive results (up to $10 \%$ to $20 \%$ savings), at least in the short term (Ueno et al., 2006; Wood and Newborough, 2003), but in the mid to long-term studies it was found that HEM S are less effective ( $V$ an Houwelingen and V an R aaij, 1989; V an Dam et al., 2010; Hutton et al., 1986).

The objective of this paper is to explore the factors influencing the medium to long-term energy saving potential of HEMS, taking a broad perspective including the design of the device (and the way it gives feedback), the human-device interactions, and the social, physical and organisational contexts in which HEMS are used and implemented. Most literature today is predominantly focused on the effectiveness of feedback, for instance by studying the relationship between different types of feedback and energy savings, or by developing novel design approaches for giving feedback 
(Froehlich et al., 2010). A few authors have explored user interactions with energy monitors (Wood and Newborough, 2003) or social contexts of use (Hargreaves, 2010). Longitudinal field research is however rare, as is research addressing these different approaches to HEM S holistically.

This paper will build on findings from literature, and will present additional insights from three case studies by the authors since 2008. The case studies are a joint cooperation between energy companies, HEMS manufacturers and the Delft University of Technology. In each case study a different HEMS was used. Focus groups, in-depth interviews, and observations led to a range of insights into how people use HEMS in daily life. The paper will conclude by presenting several new factors that contribute either positively or negatively to the medium to long-term effectiveness of HEMS.

\subsection{Method}

Three case studies were conducted with three different types of HEM S. All took place in the N etherlands. The first case study ( $V$ an Dam et al., 2010) ran between 2008 and 2009 with 189 participants, for a total of 15 months. The HEMS used was an electricity monitor that gave real-time and cumulative ( 24 hour) feedback. The quantitative data for this study was gathered by means of self-reported meter readings and four online questionnaires.

The second study dealt with ten households who implemented an energy manager in 2010. This type of HEMS gave real-time and historical feedback on the electricity consumption of individual appliances. The system worked with a touch-screen or via the participants' personal computers and could be used to manage if and when the connected appliances consumed electricity. Two months into the five-month pilot, usage data was gathered during house visits by means of qual itative, semi-structured in-depth interviews.

In the third case study a multifunctional HEM S was used, which gave historical, and for a subgroup of participants real-time, feedback on gas and electricity consumption. The device (a touch-screen) doubled as a programmable thermostat and could also provide up-to-date weather and traffic information. In total, 69 participants used this HEM S in their homes for six to 12 months. Data collection took place by means of three online questionnaires, five focus group sessions, ten in-depth interviews with households, and two usability studies (where the device and different user interfaces were tested in a lab setting).

In total, across the three case studies we could draw experiential data from 290 participants, over a period of five to 15 months. The participants were between 26 and 78 years old and had diverse educational backgrounds and family situations. In-depth qualitative data was gathered from a total of 50 unique participants. In 14 instances, participants took part in multiple sessions, giving the data extra depth and richness. A mixed-method approach was used for data analysis, involving statistical analysis of datasets as described in V an Dam et al. (2010), and qualitative analysis. For the qualitative analysis, all interviews and focus group sessions were transcribed and the datasets were analysed using a recursive abstraction approach (which involves summarising datasets to elicit insights). The findings in this paper are mainly drawn from the qualitative data analysis, using literature and the available quantitative data to substantiate the results. 


\subsection{Conceptual framework}

In order to enhance the energy saving potential of a home energy management system, it is important to improve the quality of the (long-term) interactions between the user(s) and the device. This is the central tenet of this paper. A useful framew ork for human-product interaction (Figure 1) was developed by Van K uijk (2010) and previously applied in a sustainability context by Wever et al. (2008) and adopted for this paper.

Figure 1 Framew ork for human-product interaction (with 'time' added)

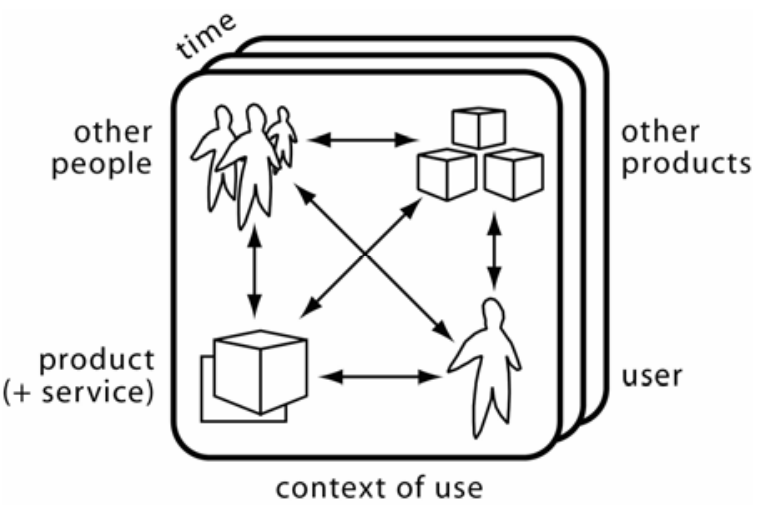

Source: After Wever et al. (2008) and V an K uijk (2010)

When applied to HEMS, this framework illustrates the interactions that take place between a user and a HEMS, and between the HEM S and other networked products. It also shows that other people can be involved in or affected by a person's HEMS use. Finally, the context of use (i.e., home context, organisational context) will be a determining factor for the overall quality of the user-device interaction. These interactions will be dealt with one by one in the following sections. Within the context surrounding the HEM S-user interaction we would like to propose the additional element of time, as this is an important factor in the use and effectiveness of HEM S.

\section{HEMS - user interaction}

Following the framework in Figure 1, this section addresses the one-on-one interaction between users and HEMS. If these devices are to fulfil their purpose, namely to help households save energy, they must be used actively, and over a longer period (several years). There are how ever different factors that impede this prolonged use.

\subsection{Malfunctioning devices}

The participants' homes were the context in which the interactions with the installed HEM S took place. This section looks at an important, but often overlooked, aspect of this context of use: a reliably functioning HEMS. The systems used in the case studies malfunctioned relatively often. Even commercially available HEMS appear to malfunction regularly. Incorrect installation, poor system capabilities, faulty hardware or software, can all have a severe effect on user perception. A s one participant explained: 
"If you get inexplicable peaks of 180 euros for gas and 150 euros for el ectricity per day it is hard to believe the rest. Y ou can't steer on it... A II these numbers, measurements, they don't mean anything anymore."

In the Netherlands, electricity suppliers and grid managers lead the implementation of HEMS, often in collaboration with commercial enterprises (that are responsible for design and production of HEMS). HEM S are increasingly offered to households via the energy suppliers, who also take care of the system's installation and maintenance. Some systems can be purchased on the market; these require consumers to self-install.

Having properly functioning HEMS is a straightforward condition that needs to be met, but this appears to be challenging for several reasons:

- The wide variety of electricity and gas meters in use today, in the Netherlands. HEM S must be able to communicate with them all. Even smart meters can be very different (i.e., they utilise different communication protocols, and some are unable to transmit real-time feedback), making it very hard to develop a generic HEM S that works reliably with all possible systems.

- The novelty of the technology used. Even though electricity monitors were introduced over three decades ago (Funk, 1978), and the first gas monitor in the N etherlands in 1982 ( $V$ an B eurden, 1982), the real technology development seems to have accelerated only in recent years with the introduction of different protocols for wireless transfer of energy data, and the possibility to give real-time data, via (interactive) displays or via users' PCs. W ith a rapidly developing technology landscape, new HEMS can be almost out-dated when they come on the market.

A nalysis of the case study data confirmed that users with faulty HEMS stopped using the devices, even after these had been repaired. Obviously, if the data cannot be trusted, people stop trying, which in effect makes HEM S useless.

\subsection{User interface}

The developers of HEMS have to combine technological know-how with user-centred design principles in order to make high-quality HEM S interfaces. It seems that for many HEMS technological challenges have dominated the development, resulting in devices with a rather 'technical' interface (see Figure 2). Many study participants expressed difficulties interpreting interfaces with numerical digits or mathematical graphs. In our third case study a participant remarked: "It is all numbers; it doesn't mean anything to me". Likewise, Kidd and Williams (2008) reported a participant in one of their studies saying "I certainly haven't used it... I certainly am not techno..."

Figure 2 Example of numerical feedback ('technical interface')

$$
\begin{array}{|ll}
\hline 351 & \text { MATT } \\
1.45 & \text { MI3/HOL }
\end{array}
$$

Only the most tech-savvy participants, we found, had little problems with such data. The design of the interface of HEM S is a subject that needs further attention. Froehlich et al. (2010, p.2004) noted this, asking: "How important is it that eco-feedback be even 
minimally interactive? What types of information and presentation mediums are most effective (e.g., graphs versus abstract ambient representations)?"

Based on our findings, part of the answer should be that interfaces with complex graphs and lots of numbers are unlikely to be acceptable to a large group of users. Some of our participants, for instance, felt more comfortable with abstract indicators (i.e., colours, size). A usability participant said: "So then those numbers are sort of meaningless...I think colours more. M ore than numbers". A nother participant used the 'clicking' sound of his current thermostat as an auditory prompt. He indicated: "If I think it's too warm in the house and I hear 'click', then I know that someone has altered the settings of the thermostat. A nd then I say: 'who touched the thermostat?"

We conclude that users are 'wired' differently and have different needs and expectations. Successful HEM S should accommodate the significant variation in users' capacities and manners of cognitively processing information by using a combination of methods to relay energy consumption data. Consideration should be given to the other senses as well, not just visual stimuli.

\subsection{One size does not fit all}

The characteristics of users and their needs and expectations influence their preferences for a specific type of HEMS. In our case studies, some participants had a preference for feedback at household level while others wanted data on each individual product (i.e., disaggregated feedback). In a focus group session, one female participant wanted to know 'everything': each detail as to the consumption of every appliance through time, while another female participant indicated that an indication of total consumption was quite enough for her. Some participants were interested in receiving nightly consumption figures because that is where they felt they could save, others did not want to be confronted with real-time consumption data. As one said: "that makes me panicky, that's not healthy". One participant was interested in a monthly or quarterly overview of his consumption to see how it changes over time, but this was "only to be viewed on my own initiative".

It is tempting to 'classify' users according to energy conservation characteristics. Liikkanen (2009) tried this, identifying three types of HEM S users: wisdom seekers, detectives and judges. Each 'type' looked for different kinds of feedback. Based on our research, we could probably add a few more types (i.e., prognostics, tab keepers, easy savers), but we feel that categorising people is too complex to discuss within this article, and apart from that, people's attitudes and usage of HEM S tend to change over time. We would like to point out that one size does not fit all, meaning that there's probably a market for a variety of HEMS that offer different approaches towards feeding back energy data. For instance, HEMS can offer aggregated feedback (household level) or disaggregated feedback (product level). For any HEMS design, however, due consideration should be given to the most likely ways the system will be implemented and used, taking into account how people learn, and enabling HEMS to evolve with the users over time.

\subsection{The energy enigma}

It is not a given that users have a (correct) conception of what a $\mathrm{kW} \mathrm{h}$ or $\mathrm{m}^{3}$ entails, nor that they know what to do with that information. Two of the 14 participants in our 
usability study had difficulties understanding $\mathrm{m}^{3}$. One said, "what does ' $\mathrm{m}$ ' ' 3 ' mean?" A second person asked: "It says here 2 uhmm... How do you measure gas again? Cubic or something?" In our first case study (V an Dam et al., 2010), the participants were required to self-report their meter readings, resulting in a high margin of error. It appeared that many participants had omitted digits, reversed the readings of day and night tariffs, or had reported data that was for example a factor 100 off from their previous readings. This seems to suggest that the conception of energy is low amongst households. Literature affirms this view: "for many people, processing complex energy information presents a formidable task" [Stern and A ronson, (1984), p.83].

Part of the challenge of HEMS is giving users a better conception of what energy entails. One possible strategy, which is often employed, is to translate energy data into monetary values. Our research findings suggest that this works for some, but (again) not for all participants. Therefore, the design of HEM S should consider the user's depth and understanding of energy. A design solution we advocate is a layered interface, which provides simple information at a quick glance and increases the depth of information in ensuing steps. For this 'first glance', simplicity is a key factor, as Fogg (2009) explains in his research on persuasive design. If it takes too much effort people will not be persuaded to act on it, or to embark on a search process.

A nother approach is to see HEM S not as the ultimate solution, but as part of a system, where users are stimulated to enter a search process that leads to other media or people for further analysis and in-depth information. Stern and A ronson (1984) advocate a 'full court press', saying: "feedback is more likely to be effective if given as part of a program in which the energy user is an active participant rather than simply being a passive recipient - even when information is offered about how to interpret the feedback" (p.88).

\subsection{The baseline check}

As users interact with HEMS, new behaviours develop. HEMS becomes embedded in people's lives and routines start to surface. In the first case study, it appeared that the most popular routine was to check the electricity consumption before going to bed, affirming that the house was at its baseline energy consumption. The baseline energy consumption is the amount of electricity a household consumes when none of the appliances are in active use. In most homes, electricity is consumed 24/7, for instance to keep the ventilation system and refrigerator going and to power the products that are in standby mode. This routine, which we called 'the baseline check', was also noted in Kidd and W illiams (2008) who quoted a user:

\footnotetext{
"there were times sitting in bed, turned the light out and then try to get the little light (on the monitor) to come on so we could read it in the dark - 'yea, we've dropped! Night night, darling!"
}

In our second case study, a participant indicated he would like a small screen in his bedroom so he could check and turn off appliances that had accidentally been left on while he was al ready in bed.

For HEMS to be used effectively over time, they should become embedded into people's daily routines. Understanding which daily routines emerge from the use of a certain type of HEMS can be important information for designers, as it helps them tailor the system to those preferred use situations. 


\subsection{Obsoleteness}

In our first case study, we found that a significant user group had incorporated the electricity monitor into their daily routines and checked it habitually. An even larger group however (just over half of the participants) had stopped using the electricity monitor, with the majority opting to stop using and giving back the device after the first four months of the study. We found that when people were beginning to lose interest, relatively small triggers like a (temporary) malfunction or even the need to change the battery could be the 'last straw'. One lady using the energy manager in the second study said: "I believe my husband has pulled the plug [of the device] out again... so then I thought; I'm really giving up". U eno et al. (2006) found a significant decrease in the interactions with the energy monitor that was used in their nine month study, indicating a similar drop-off rate. There is obviously a risk that HEM S become obsolete long before their technical lifetime is over. Studying how HEM S are used (and disused) in the short and long term and implementing these findings in the design to make them fit better, might reduce those risks.

\subsection{It is my 'thing'}

In talking to HEM S users, it surfaced that the device is often the 'pet' tool of one person in the family. A s one person using the energy manager said: " $Y$ es I am the user of it, yes, not even $99 \%$ but 100\%". Figure 3 shows that the majority of households had one main user, and Figure 4 shows that this user was generally male.

Figure 3 Number of main users per household after 15 months $(N=93)$

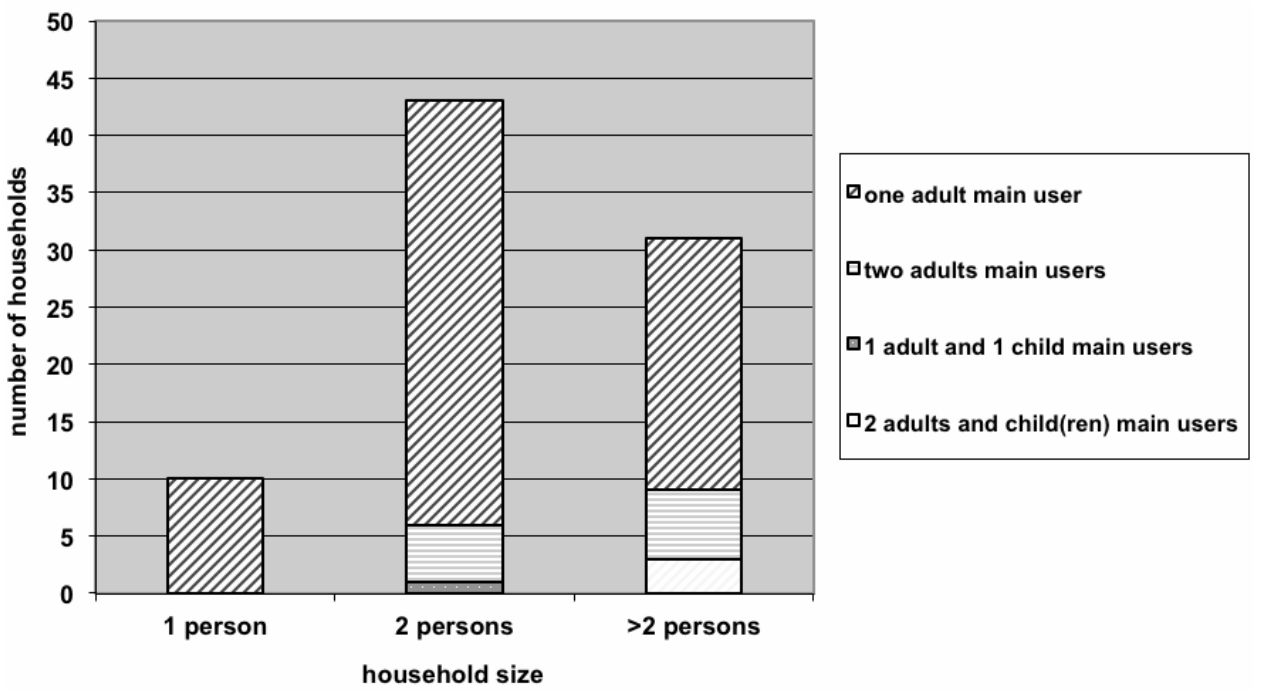


Figure 4 Gender of the main user(s) ( $N=93)$

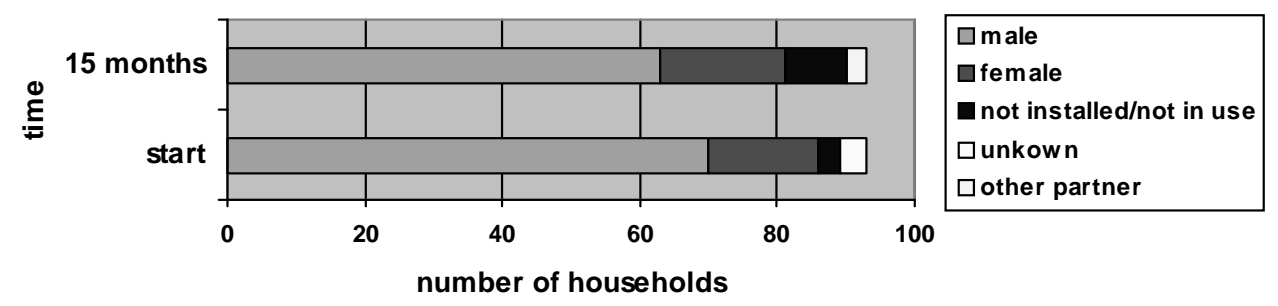

The findings of Hargreaves' (2010) interviews with 15 participants were in line with our one-main-male-user finding. It is however possible that different HEMS appeal to different genders and that one of the areas to be addressed is how to make HEMS more appealing to women. Because it is not always the feedback as such that is unappealing to women but sometimes the way in which it is presented or designed:
“M y wife doesn't like it as much as just the old thing, which had a single big number on it, because there are too many things on here for her to look at. She doesn't understand it really. She understands a tick and a cross so that's okay." (Hargreaves, 2010)

In the second case study, a female signed up with the motivation:

"Well, I am more energy conscious, and they [points at her husband and 2 daughters] aren't... I don't understand anything at all about it [the HEM S], but I am also not technical for the rest. Only I was a bit like, let's see what our phantom loads are."

It is worth pursuing whether HEMS can be designed to appeal to more members of the household.

\section{Interaction between users and other people}

This section will look at the way the main HEMS user relates to other members of household (see Figure 1), as this may have an important bearing on the effectiveness of HEMS usage. Also, the social context of households will be taken into account, where households compare themselves to other households.

\subsection{Sustainability and/or cost advocates}

Often, one adult in a household holds sustainability dearest to his/her heart. This person is the family catalyst concerning sustainability. The tactics vary, but regularly this person will 'badger' other members of the household concerning the length of their showers, their forgetfulness in turning off lights or appliances, etc. For a number of households this is not just about sustainability but also (or only) about cost management (Figure 5).

Some of the sustainability/cost advocates who tested an energy manager indicated they now feel better equipped to this task. One participant said:

"M y children used to shower every other day. But nowadays they all - except for me - shower every day. And sometimes they take long showers, and then the door is locked, so then I have to knock on the door once in a while. Like: 
'hurry up a bit!'. How much that costs, I don't know exactly. But now I could quickly run down the stairs to look. A nd then I can also show it..."

A female participant said she hoped her husband would use it and change his behaviour. Five months later she reported that her son and husband were starting to pay attention to not leaving lights and the computer on, but commenting that "I still have to switch the lights off after them once in a while". A nother participant indicated that their "consumption had become easier to discuss with the family members". Whether the main HEM S user in households is always the same person as the sustainability or cost advocate is an interesting question for further research.

Figure 5 Which household member is most interested in sustainability and/or pays the most attention to costs $(N=53)$

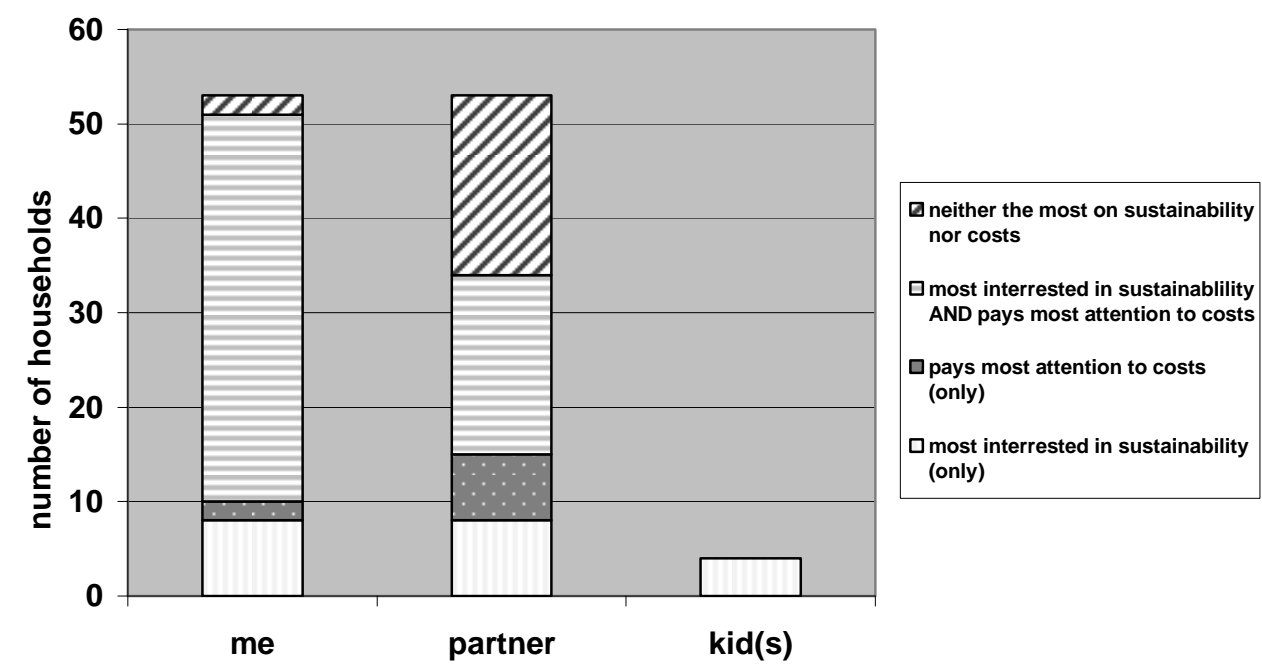

Outside these case studies, mention has also been made that users apply a HEMS as 'badgering tool':
"I mean some nights I can come home from work and the whole house is lit up like Blackpool Tower - the computer's on, the telly is on, the radio's on in here and there's nobody in the house! That used to drive me up the wall but they are now starting to think. I've been badgering them and I've been flashing that meter in their faces!" (Kidd and Williams, 2008)

Hargreaves (2010) approaches these 'family negotiations' as a limitation, warning for a potential increase in conflicts and stressing the need to consider the complex social context in which HEMS are implemented. HEMS should therefore aim at opening a positive dialogue between members of households and serve as tool in helping identify, and alter specific behaviours of members of household.

\subsection{The boomerang effect}

Moving to the broader social context surrounding households, households need a reference for their total energy consumption. But this needs to be tailored to different household types. Several participants in our case studies indicated they did not know if 
the energy consumption displayed on their HEMS was abnormal or not, and some jumped to (unsupported) conclusions. In the first case study one participant, with an annual consumption of 4,110 kWh (which is slightly above average) and a family of four, concluded after two months that: "the electricity monitor confirms that we are doing well. Further savings would barely be possible in our household". It might therefore be useful to give households a norm to compare themselves to. However, Schultz et al. (2007) warn of the boomerang effect: the phenomenon where households increase their energy consumption, instead of decreasing it, because they are below the norm (the norm being the average neighbourhood energy consumption). Researchers disagree on how to prevent a boomerang effect from happening. Schultz et al. (2007) claim that giving people normative as well as injunctive feedback (approving or disapproving certain behaviour) eliminated the boomerang effect. Ayres et al. (2009) however, did find the boomerang effect for low energy consumers in both their studies even though both descriptive as well as injunctive norms were used. The overall energy savings in these studies were however still positive. This indicates than when using comparative feedback, care should be given to preventing the boomerang effect. This could be done either by only giving high-energy consumers comparative feedback, thus creating tailored HEM S, or by setting different norms for different use groups and adjusting these through time.

\section{Interactions between HEMS and other products/users}

HEMS have a mediating role, they provide people with a (visual) representation of energy consumption, and help them mentally interpret the actual energy (or monetary) figures and perceive the energy consumption of other products. Ihde (1990) calls this relationship between users and products a hermeneutic relationship. The HEMS-user interaction cannot be seen separate from the relationship between HEMS and other symbiotic products or users and other products. This next section will centre on this part of the conceptual framework (Figure 1): the triangular interaction between HEM S, users and other products.

\subsection{Cause and effect}

Understanding energy consumption is hard for many people. This makes it difficult to relate the feedback given by HEMS to real actions. Plain facts on energy consumption bring knowledge but not understanding. When a household knows which actions cause a large spike in energy consumption, it is far easier to act upon this. Energy consumption varies significantly throughout the week, making an average daily consumption figure relatively useless. One participant in the third case study said about the cumulative total of the last 24 hours: "it is useless for me". A s Darby (2010) noted, extrapolating daily or weekly consumption to expected monthly or yearly consumption can lead to highly erratic estimates, which users can find hard to trust and difficult to act upon. In their design, HEMS should strive to make the consequences of the users' actions clear, and not resort to useless statistics. Admittedly, achieving useful figures and statistics can be challenging and will require user testing. 


\subsection{How to manage the energy manager}

Certain types of HEMS (called energy managers) can help people control if and when appliances use energy and circumvent the complexity of behaviour change, for instance through scheduled timers. Timers are however not always a good solution. For one, households' schedules fluctuate throughout the week. With increasing household size, the household dynamics and rhythms of daily life become increasingly complex. In the second case study, this led to members of households (permanently) bypassing the system when it was not in sync with their lifestyle, thereby annihilating the savings. A Iso, households with programmable thermostats appear to use more energy than those with a manual thermostat (Guerra Santin et al., 2009).

In general, most households have little knowledge of the workings of their appliances to successfully use an energy manager. This can lead to appliances being switched off that should not be (e.g., for safety reasons) or appliances that are left 'on' unnecessarily. Two of our case study participants put a timer on their refrigerators to make them turn off during the night; several participants did not know their appliances or gadgets (e.g., game consoles) were still consuming power even though they had switched them 'off'; and one participant never shut down his modem for fear of receiving a blacklisted IP address. The difficulty for many people is not turning on their devices; the problem is turning them off (or not knowing when or how to do this correctly). Designers should be aware of this when designing household appliances and consumer electronics.

Households can find themselves 'locked-in' by their own appliances. Jackson (2005) describes lock-in as "far from being able to exercise deliberative choice about what to consume and what not to consume". Two users in the third case study stated that certain energy inefficient behaviours were not of their own choice. While they themselves did not see the necessity of heating the whole house or even preferred not to, they were 'forced' by their heater. Either their installer had prescribed the need to heat a majority of rooms for their heater to function properly or the heater had actually given an error code because it was heating too few rooms. A third user was in the process of changing his lights to energy efficient alternatives but was unable to do so as his halogen lights "were not replaceable by an energy efficient version".

This teaches us that even highly motivated people may find it hard to change their energy consumption behaviour, due to their (busy) lifestyles, lack of knowledge on how and when to turn off their products and choices from the past that hinder change.

\section{HEMS effectiveness}

The usability framework of Figure 1 offers a practical model for describing our observations regarding the daily usage of HEM S in three different case studies. This final section will address the cumulative user experience over time (as indicated in the 'timeline' in Figure 1). More precisely, we will examine the relationship between the usability of HEM S and their effectiveness. Effectiveness is defined as the extent to which users can maintain net energy savings over prolonged periods. 


\title{
5.1 Short term effects
}

The overall effectiveness of HEMS in mid to long term case studies is indecisive ( $V$ an Houwelingen and V an Raaij, 1989; Mountain, 2006) or shows a negative trend ( $V$ an Dam et al., 2010). To date, the effectiveness of HEM S seems mostly short lived. We have already discussed that HEMS are not useful for all households, and not all households want to, or manage to, reduce energy consumption. It is therefore questionable whether implementing HEMS 'en masse' will contribute to overall energy efficiency. In fact, it might result in an overall decrease of the total HEMS effectiveness. Careful consideration is therefore needed as to which user groups will be targeted.

\subsection{Process of change}

A number of participants in the case studies indicated that acquiring an HEMS was part of a longer process of change in which they were 'growing' towards sustainability. One participant in the second study explained:

\begin{abstract}
"I think that in part I did become more conscious when I started working at the ASN bank [sustainable bank in the Netherlands (eds.)]. Also about all the possibilities there are [to be sustainable]. Y ou don't realize all those options in the beginning."
\end{abstract}

He indicated that through the energy manager he had received more insight into the standby use of appliances and turned them off at night, adding that: "[It] was also pointed out a bit by my colleagues in the past", and commented further on the persuasive social influence his colleagues had on him. A second participant responded in a similar way. He said: "that I became more conscious is 4 years ago, I think approximately". He was now implementing flip switches and timers throughout the home. When asked about the reason he explained he was inspired by an 'eco-minded' colleague. Sometimes there is a direct trigger, like a participant's bill: "In the last electricity bill [half a year before] it turned out that we used about one and a half or two times the national average". He continued: "In first instance I messed around a bit with energy meters and that sort of thing" after which he signed up for the case study. Two months into the study he purchased solar panels. Coincidentally there was a price incentive offered by his municipality that influenced his decision, which again gives an indication that HEMS should be seen as part of a larger scheme.

Woodruff et al. (2008) found that for the 35 homes they interviewed, sustainability was often a progressive development over many years. While for some sustainability is a philosophy that permeates their whole life, certain phases in life or financial positions can assist or inhibit this process. As one focus group participant told, his electricity consumption dropped from 5,500 to $3,200 \mathrm{kWh}$ when his two sons moved out. This aspect needs to be examined in more detail, in order to understand the role HEMS can play in aiding people in this change process. Measuring the exact effects of HEMS however, becomes far more complicated as it can be part of a chain reaction and permeates into other areas of life. The examples above also show that social dynamics are an important aspect to consider. 


\subsection{Net impact of HEMS}

Consideration should be given to the overall life cycle impact of HEMS and not only to the savings that can be achieved. In other words, a trade-off needs to be made between the energy needed for production, use and disposal of the device versus the amount of energy saved by using it. When the savings achieved through HEM S are only sustained for a short period, it is hard (or even impossible) to break even with the amount of energy invested. An assessment of the cumulative energy demand (CED) can be a useful tool here. In light of the uncertain long-term effects of HEMS it can be argued that these devices should not be developed as stand-alone products, but should be integrated in existing products instead. Care should however be taken that the simplicity and accessibility of the feedback is maintained.

\subsection{Smart grid future}

A smart grid system allows for two-way communication between utilities and consumers. Smart grids focus on peaking shifting, e.g., through dynamic pricing schemes. This process employs smart meters and HEMS that provide real-time pricing, which gives consumers the incentive to reduce electricity use during high-priced peak periods. Research from 1987 implementing HEMS with pricing schemes has shown that the overall consumption is not reduced but rather shifts to periods with cheaper electricity (Sexton et al., 1987), implying that smart grid systems mainly lead to different energy usage behaviour (and not necessarily to energy conservation). The environmental benefit of smart grids is the creation of more evenly distributed grid-loads, but such societal benefits are relatively abstract for individual households and difficult to communicate. In a smart grid system, HEM S will be indispensible for giving users insight in the energy pricing schemes. They may also offer some level of control over smart appliances. However, the amount of user control and the influence this has on energy consumption needs further study.

\section{Conclusions}

As stated in the introduction, the aim of this paper was to present a number of insights useful to the design and implementation of HEMS. By using the conceptual framework on human-product interaction, a number of important areas have been highlighted that have received little attention in literature reporting on the effectiveness of HEMS feedback. It has been shown that studying the HEM S-user interaction and how this changes over time can give a wealth of knowledge that can contribute to better design and implementation. Additionally, family dynamics and the social context are a notable factor in the acceptance of HEM S and the achievable savings. The paper has also looked at the triangular interaction between HEMS and its user and other symbiotic products showing that these intertwine with the achievable effects of HEM S.

Finally, the paper concluded that the 'effects' of HEMS should be placed in a wider context. Effects are far more complex and harder to define than the just direct outcome on energy consumption. It is important to consider the entire life cycle and the embedded energy. Effects also need to include a time factor and the scale of implementation: how 
long HEM S are used, which (kinds of) households manage to save energy and what the 'durability' of the achieved savings is.

Some of the insights need to be further substantiated by means of quantitative data. How often do HEMS become obsolete prematurely, at what point in time does this happen and what are the causes and effects? How can feedback be made more understandable and appealing to (other) members of household and how can HEMS positively involve all members in reducing energy consumption? How detrimental are defective functioning and technical interfaces to energy savings?

In answering these questions, longitudinal research with an interdisciplinary character through close collaboration between social sciences, the human-computer interaction $(\mathrm{HCl})$ community, energy suppliers, designers and developers, as well as HEMS users, is essential. One possible case study could be to test a number of different HEMS (interfaces) simultaneously using a mixed method approach. A nother is to study the effects of implementing HEMS as part of a range of interventions and incentives to reduce consumption (a 'full court press' approach). Thirdly, experience sampling (a technique to make participants record temporal things like feelings in the moment) could prove to be a relevant approach to come to a better understanding of which household member(s) is/are using the HEM S and how, when and for what reason, so that HEM S can be better tailored to these situations.

There is still significant progress to be made in the development and implementation of HEMS. But by taking the areas shown in this paper into consideration and studying them further, the effectiveness of HEM S will improve.

\section{References}

Ayres, I., Raseman, S. and Shih, A. (2009) 'Evidence from two large field experiments that peer comparison feedback can reduce residential energy usage', NBER Working Paper Series, $N$ ational B ureau of Economic R esearch, Cambridge, M ass., USA .

Darby, S. (2010) 'Smart metering: what potential for householder engagement?', Building Research \& Information, Vol. 38, No. 5, pp.442-457.

Fogg, B.J. (2009) 'A behavior model for persuasive design', Persuasive 2009, The 4th International Conference on Persuasive Technology, Claremont, California, USA .

Froehlich, J., Findlater, L. and Landay, J. (2010) 'The design of eco-feedback technology', CHI 2010, 10-15 A pril 2010, A CM , A tlanta, Georgia, USA, pp.1999-2008.

Funk, L.L. (1978) Energy Monitoring Device, US patent application, Hillsborough, NC.

Guerra Santin, O., Itard, L. and Visscher, H. (2009) 'The effect of occupancy and building characteristics on energy use for space and water heating in Dutch residential stock', Energy and Buildings, V ol. 41, No. 11, pp.1223-1232.

Hargreaves, T. (2010) 'The visible energy trial: insights from qualitative interviews', Tyndall Centre for Climate Change R esearch.

Hutton, R.B., M auser, G.A., Filiatrault, P. and A htola, O.T. (1986) 'Effects of cost-related feedback on consumer knowledge and consumption behaviour: a field experimental approach', Journal of Consumer Research, V ol. 13, N 0. 3, pp.327-336.

Inde, D. (1990) Technology and the Lifeworld from Garden to Earth, Indiana University Press, Bloomington, Indiana.

Jackson, T. (2005) 'M otivating sustainable consumption - a review of models of consumer behaviour and behavioural change', A report to the Sustainable Development Research N etwork, Policy Studies Institute, L ondon. 
Kidd, A . and W illiams, P. (2008) The Talybont Trial, Exploring the Psychology of Smart Meters, The Prospectory, B recon, Wales.

Liikkanen, L. (2009) 'Extreme-user approach and the design of energy feedback systems', International Conference on Energy Efficiency in Domestic Appliances and Lighting 2009 (EEDAL'09), B erlin, Germany.

M ountain, D. (2006) The Impact of Real-Time Feedback on Residential Electricity Consumption: The Hydro One Pilot, M ountain Economic Consulting and A ssociates, Inc., O ntario.

Schultz, P., Nolan, J., Cialdini, R., Goldstein, N. and Griskevicius, V. (2007) 'The constructive, destructive, and reconstructive power of social norms', Psychological Science, Vol. 18, No. 5, p.429.

Sexton, R.J ., J ohnson, N.B . and K onakayama, A . (1987) 'Consumer response to continuous-display electricity-use monitors in a time-of-use pricing experiment', The Journal of Consumer Research, Vol. 14, No. 1, pp.55-62.

Stern, P. and Aronson, E.E. (1984) Energy Use: The Human Dimension, W.H. Freeman and Company, New Y ork.

Ueno, T., Inada, R., Saeki, O. and Tsuji, K. (2006) 'Effectiveness of an energy-consumption information system for residential buildings', Applied Energy, V ol. 83, N 0. 8, pp.868-883.

Van Beurden, K. (1982) 'Residential gas consumption indicator', Gas, February, Vol. 102, pp.58-64, A peldoorn, Netherlands.

Van Dam, S.S., B akker, C.A. and V an Hal, J.D.M . (2010) 'Home energy monitors: impact over the medium-term', Building Research \& Information, V ol. 38, No. 5, pp.458-469.

$V$ an Houwelingen, J.H. and V an Raaij, W.F. (1989) 'The effect of goal-setting and daily electronic feedback on in-home energy use', The Journal of Consumer Research, V ol. 16, No. 1, pp.98-105.

Van Kuijk, J. (2010) 'M anaging product usability; how companies deal with usability in the development of electronic consumer products', PhD thesis, Delft University of Technology, The N etherlands.

Wever, R., Van Kuijk, J. and Boks, C. (2008) 'User-centred design for sustainable behaviour', International Journal of Sustainable Engineering, V ol. 1, N o. 1, pp.9-20.

Wood, G. and Newborough, M. (2003) 'Dynamic energy-consumption indicators for domestic appliances: environment, behaviour and design', Energy and Buildings, Vol. 35, No. 8, pp.821-841.

Woodruff, A., Hasbrouck, J. and A ugustin, S. (2008) 'A bright green perspective on sustainable choices', Conference on Human Factors in Computing Systems, 2008 Florence, Italy, ACM, N ew Y ork, NY, USA, pp.313-322. 